\title{
A proof for Berge's Dual Conjecture for Bipartite Digraphs
}

\author{
Caroline Aparecida de Paula Silva ${ }^{1}$, Cândida Nunes da Silva ${ }^{1}$, Orlando Lee $^{2 \dagger}$ \\ ${ }^{1}$ Departament of Computing - Federal University of São Carlos (DComp-UFSCar) \\ Rod. João Leme dos Santos km 110 - SP-264, CEP 18052-780, Sorocaba - SP - Brazil \\ ${ }^{2}$ Institute of Computing - University of Campinas (IC-UNICAMP) \\ Av. Albert Einstein, 1251, Cidade Universitária, CEP 13083-852, Campinas - SP - Brazil \\ caroline.silva@dcomp.sor.ufscar.br, candida@ufscar.br, lee@ic.unicamp.br
}

\begin{abstract}
Given a (vertex)-coloring $\mathcal{C}=\left\{C_{1}, C_{2}, \ldots C_{m}\right\}$ of a digraph $D$ and a positive integer $k$, the $k$-norm of $C$ is defined as $|\mathcal{C}|_{k}=\sum_{i=1}^{m} \min \left\{\left|C_{i}\right|, k\right\}$. A coloring $\mathcal{C}$ is $k$-optimal if its $k$-norm $|\mathcal{C}|_{k}$ is minimum over all colorings. $A$ (path) $k$-pack $\mathcal{P}^{k}$ is a collection of at most $k$ vertex-disjoint paths. A coloring $\mathcal{C}$ and a $k$-pack $\mathcal{P}^{k}$ are orthogonal if each color class intersects as many paths as possible in $\mathcal{P}^{k}$, that is, if $\left|C_{i}\right| \geq k,\left|C_{i} \cap P_{j}\right|=1$ for every path $P_{j} \in \mathcal{P}^{k}$, otherwise each vertex of $C_{i}$ lies in a different path of $\mathcal{P}^{k}$. In 1982, Berge conjectured that for every $k$-optimal coloring $\mathcal{C}$ there is a $k$-pack $\mathcal{P}^{k}$ orthogonal to $\mathcal{C}$. This conjecture is false for arbitrary digraphs, having a counterexample with odd cycle. In this paper we prove this conjecture for bipartite digraphs. In addition we show that the conjecture cannot hold for perfect graphs by exhibiting a counterexample.
\end{abstract}

\section{Introduction}

Given a graph $G$, we denote its set of vertices by $V(G)$ and its set of edges by $E(G)$. The order of $G$ is the cardinality of $V(G)$, denoted by $n$. Given two vertices $u, v \in$ $V(G)$, we say $u$ and $v$ are adjacent if $(u, v) \in E(G)$ and non-adjacent otherwise. An independent (vertex)-set in $G$ is a set of pairwise non-adjacent vertices. We denote as $\alpha(G)$ the cardinality of a maximum independent set of a graph $G$. A vertex cover of a graph $G$ a subset $V^{\prime}$ of $V(G)$ such that every edge $e \in E(G)$ has at least one end in $V^{\prime}$. We denote the cardinality of the minimum vertex cover as $\tau(G)$. In the celebrated paper [Gallai 1959] the existence of a relation between the cardinalities of a maximum independent set and a minimum vertex cover was observed for graphs in general, as stated below:

Theorem 1 (Gallai). For every graph $G$, the equality $\alpha(G)+\tau(G)=n$ holds.

We say that $G^{\prime}$ is a subgraph of a graph $G$ if $V\left(G^{\prime}\right) \subseteq V(G)$ and $E\left(G^{\prime}\right) \subseteq E(G)$. Given a subset $S \subseteq V(G)$, we define the subgraph of $G$ induced by $S$, denoted $G[S]$, as the subgraph having $S$ as its set of vertices and such that whenever $u v$ is an edge of $G$ with both ends in $S$, then $u v$ is also an edge of $G[S]$.

A graph $G$ is $(X, Y)$-bipartite if $V(G)=X \cup Y, X \cap Y=\emptyset$ and the induced subgraphs $G[X]$ and $G[Y]$ are independent sets. A matching in a graph $G$ is a subset

\footnotetext{
* Support by FAPESP (grant 2018/11462-9) and CNPq (PIBIC-UFSCar program).

${ }^{\dagger}$ Support by FAPESP (grant 2015/11937-9) and CNPq (grants 303766/2018-2 and 425340/2016-3). ORCID: 0000-0004-4462-3325.
} 
$M \in E(G)$ where, for every vertex $v \in V(G)$, at most one arc of $M$ is incident with $v$. We denote the cardinality of a maximum matching by $\alpha^{\prime}(G)$. Another celebrated theorem proved in paper [König 1931] establishes an equivalence between cardinalities of the maximum matching and of the minimum vertex cover in bipartite graphs:

Theorem 2 (König). If $G$ is a bipartite graph, then $\tau(G)=\alpha^{\prime}(G)$.

We deduce, from these two theorems, the following Corollary:

Corollary 1. If $G$ is a bipartite graph then $\alpha^{\prime}(G)+\alpha(G)=n$.

Given a digraph $D$, we denote its set of vertices by $V(D)$ and its set of arcs by $A(D)$. The order of $D$ is the cardinality of $V(D)$, also denoted by $n$. Given two vertices $u, v \in V(D)$, we say $u$ and $v$ are adjacent if either $(u, v) \in A(D)$ or $(u, v) \in A(D)$ and non-adjacent otherwise. This paper is focused on the study of properties of digraphs. However, in order to state the problem and present our contribution, we need concepts that are usually defined for graphs, such as the ones already defined. In order to avoid confusion and also frequent repetitions of definitions, we will establish that, whenever a concept defined for graphs is applied to a digraph $D$, we are actually applying that concept to the underlying undirected graph $G(D)$ of $D$, i. e., the graph obtained from $D$ by identifying $V(G)=V(D)$ and interpreting every ordered pair $(u, v) \in A(D)$ as an unordered pair $(u, v) \in E(G)$.

A path $P=\left(v_{1}, v_{2}, \ldots, v_{p}\right)$ in a digraph $D$ is a sequence of vertices such that arc $\left(v_{i}, v_{i+1}\right) \in A(D)$ for $1 \leq i<p$. The cardinality of a path $P$, denoted as $|P|$, is the number of vertices $p$ in the sequence, which is an unusual definition in graph theory, since most authors define cardinality of a path as being its number of edges. We denote by $\lambda(D)$ the cardinality of a maximum path in $D$. A path $P$ is called trivial if $|P|=1$ and hamiltonian if $|P|=n$.

A tournament in a digraph $D$ is a subdigraph $D^{\prime}$ such that for every pair of vertices $x, y \in V\left(D^{\prime}\right), x$ and $y$ are adjacent. Paper [Rédei 1934] presents an important property of a tournament.

Theorem 3 (Rédei). Every tournament has a hamiltonian path.

A path partition $\mathcal{P}$ is a collection of vertex-disjoint paths of $D$ such that $\bigcup\{V(P): P \in \mathcal{P}\}=V(D)$. We denote by $\pi(D)$ the size of an optimum path partition of a digraph $D$, that is, a path partition that covers the vertex set of the digraph using the smallest possible number of paths. An $m$-(vertex)-coloring (or simply a coloring) $\mathcal{C}=\left\{C_{1}, C_{2}, \ldots, C_{m}\right\}$ is a collection of disjoint independent sets, also called color classes, where $\bigcup\left\{C_{i}: C_{i} \in \mathcal{C}\right\}=V(D)$. The chromatic number of $D$, denoted by $\chi(D)$, is equal to the size of an optimal coloring of $D$.

\subsection{Path Partitions and Stable Sets}

Dilworth was the pioneer in the study of the relation between minimal path partition and maximum independent sets having proved the following theorem in paper [Dilworth 1950]:

Theorem 4 (Dilworth). Every acyclic and transitive digraph $D$ satisfies $\pi(D)=\alpha(D)$.

Later, in paper [Gallai and Milgram 1960] the authors showed that the size of the minimum path partition $\pi(D)$, is less than or equal to the size of the maximum independent set $\alpha(D)$. 
Theorem 5 (Gallai-Milgram). Every digraph D satisfies $\pi(D) \leq \alpha(D)$.

Few decades later, Linial tried to investigate ways to generalize Gallai-Milgram's Theorem. To do so, he tried to associate a path partition with a collection of $k$ independent sets for a fixed integer and positive $k$. This idea demanded the definition of a new metric of minimality of path partitions and maximality of collections of independent sets.

A partial $k$-coloring of a digraph $D$ is an assignment of $k$ colors to a subset of its vertices where adjacent vertices must have distinct colors. It implies that in any partial $k$-coloring of a graph, the set of vertices having the same color is an independent set. The weight of a partial $k$-coloring is the total number of colored vertices. A partial $k$-coloring is maximum if there is no other partial $k$-coloring in which more vertices are colored. We denote by $\alpha_{k}(D)$ the weight of a maximum partial $k$-coloring. Figure 1a shows a digraph $D$ and a coloring using four colors (white, light gray, dark gray and black). In fact $\chi(D)=4$, but since the coloring presented is such that there is a unique white vertex, all but one vertex can be colored with 3 colors, i. e., $\alpha_{3}(D)=7$.

The $k$-norm of a path partition $\mathcal{P}$ is denoted by $|\mathcal{P}|_{k}$ and defined in the following way:

$$
|\mathcal{P}|_{k}=\sum_{P \in \mathcal{P}} \min \{|P|, k\} .
$$

A path partition that minimizes the $k$-norm of a digraph is called $k$-optimum. The $k$-norm of a $k$-optimum path partition of a digraph $D$ is denoted by $\pi_{k}(D)$. The path partition $\mathcal{P}_{3}=\left\{P_{1}, P_{2}, P_{3}\right\}$ depicted in Figure 1a is 3-optimum, thus $\pi_{3}(D)=3+3+1=7$.

Based on the definitions of $k$-norm of a $k$-optimum path partition and weight of a maximum partial $k$-coloring, in paper [Linial 1981] the author proposed the following conjecture, which later became known as the Weak Path Partition Conjecture:

Conjecture 1 (Linial). Let $D$ be a digraph and $k$ a positive integer. Then,

$$
\pi_{k}(D) \leq \alpha_{k}(D)
$$

It is not hard to see that when $k=1$ Linial's Conjecture is exactly GallaiMilgram's Theorem; therefore valid. Verify that, for the digraph $D$ depicted in Figure 1a, $\pi_{4}(D)=|V(D)|=\alpha_{4}(D), \pi_{3}(D)=\alpha_{3}(D)$ according to our previous discussion, and $5=\pi_{2}(D)<\alpha_{2}(D)=6$.

Almost concurrently, Berge, who was also seeking for a generalization of GallaiMilgram's Theorem, proposed a different conjecture. Berge introduced the concept of orthogonality between path partitions and collections of disjoint independent sets of a digraph in order to propose his conjecture. We say that a path partition $\mathcal{P}$ and a partial $k$ coloring $\mathcal{C}$, for a positive integer $k$, are orthogonal if each path of $\mathcal{P}$ meets the maximum number of colors in $\mathcal{C}$, that is, if a path has cardinality less than $k$, then all its vertices must have different colors, otherwise, there is at least one vertex of each of the $k$ colors in the path. The statement of Berge's Conjecture, presented in paper [Berge 1982b] is as follows:

Conjecture 2 (Berge). Let $D$ be digraph and $k$ be a positive integer. Then, for every $k$-optimum path partition $\mathcal{P}$ of $D$ there is a partial $k$-coloring orthogonal to $\mathcal{P}$.

As well as Linial's Conjecture, Berge's Conjecture also generalizes GallaiMilgram's Theorem. However, instead of only trying to generalize the statement of the 
theorem, Berge sought to generalize the orthogonality concept, which already appeared in the proof of Gallai-Milgram's Theorem. It is not hard to see that Berge's Conjecture implies Linial's Conjecture. Thus, Berge's Conjecture is known as Strong Path Partition Conjecture. In the same paper where Berge proposed the conjecture [Berge 1982b], he showed that the conjecture holds for some classes of digraphs, including the bipartite digraphs. The partial 3-coloring for the digraph of Figure 1a composed by color classes black, dark gray and light gray is an example of a partial 3-coloring orthogonal to the 3-optimum path partition $\mathcal{P}_{3}=\left\{P_{1}, P_{2}, P_{3}\right\}$.

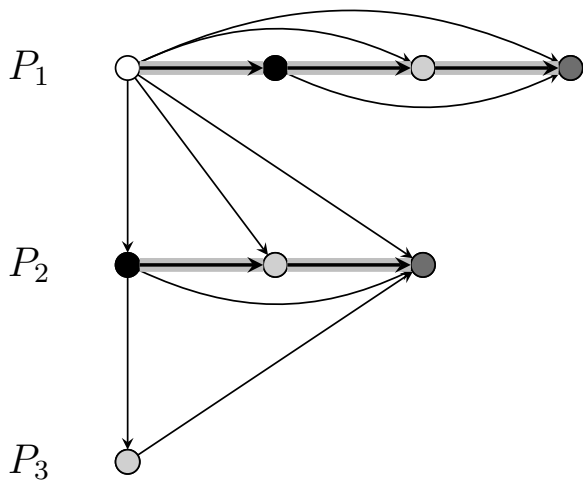

(a) 3-optimum path partition and a maximum partial 3coloring.

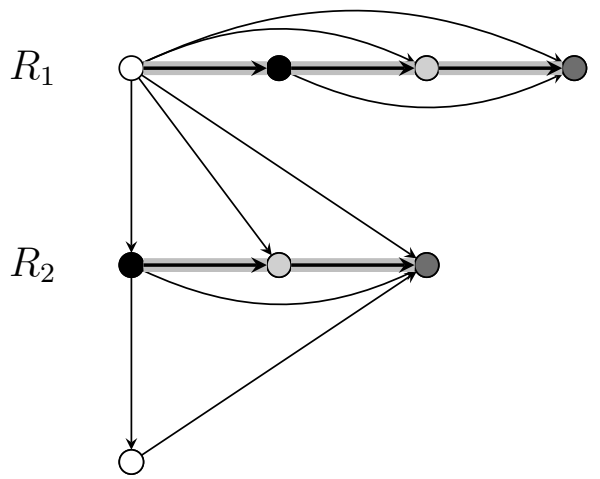

(b) 2-optimum coloring and a maximum 2-pack.

Figure 1. Example of colorings and path partitions

\subsection{Dual Relation}

For each one of the theorems that relates a path partition and a collection of independent sets, there is an analogue theorem where the roles of paths and independent sets are changed. This analogy between the two concepts is called duality and in this section we will show what is known about the dual problems.

The first theorem proved for a dual relation dualizes Gallai-Milgram's Theorem. It is known as Gallai-Roy's Theorem and it shows that the size of a minimum independent set partition (optimum coloring) is less than or equal to the size of the longest path of $D$. Theorem 6 (Gallai-Roy). Every digraph D satisfies $\chi(D) \leq \lambda(D)$.

Roy and Gallai gave independent proofs for this Theorem in papers [Roy 1967] and [Gallai 1968]

Shortly after Gallai-Roy's Theorem, but more than two decades after Dilworth's Theorem, the dual version of Dilworth's Theorem was proved in paper [Mirsky 1971]. Theorem 7 (Mirsky). Every acyclic and transitive digraph $D$ satisfies $\lambda(D)=\chi(D)$.

In order to dualize Linial's Conjecture, it is necessary to redefine the metrics now associated to a positive integer $k$. Given a coloring $\mathcal{C}=\left\{C_{1}, C_{2}, \ldots, C_{m}\right\}$ and a positive integer $k$, the $k$-norm of $\mathcal{C}$ is defined by

$$
|\mathcal{C}|_{k}=\sum_{i=1}^{m} \min \left\{\left|C_{i}\right|, k\right\} .
$$


A coloring $\mathcal{C}$ of $D$ is $k$-optimum if there is no coloring $\mathcal{B}$ of $D$ such that $|\mathcal{B}|_{k}<$ $|\mathcal{C}|_{k}$. We denote by $\chi_{k}(D)$ the $k$-norm of a $k$-optimum coloring of $D$. Figure 1 b shows that the digraph $D$ we have previously analyzed also has a coloring using four colors such that each color class has two vertices; thus $\chi_{2}(D)=8=\chi_{3}(D)=\chi_{4}(D)$.

A $k$-pack $\mathcal{P}^{k}$ is a family of, at most, $k$ vertex-disjoint paths. Let $V\left(\mathcal{P}^{k}\right)=$ $\bigcup\{\{V(P): P \in \mathcal{P}\}\}$, the weight of a $k$-pack is defined by $\left\|\mathcal{P}^{k}\right\|=\left|V\left(P^{k}\right)\right|$. A $k$-pack $\mathcal{P}^{k}$ of a digraph $D$ is optimum if there is no $k$-pack $\mathcal{Q}^{k}$ in $D$ such that $\left\|\mathcal{Q}^{k}\right\|>\left\|\mathcal{P}^{k}\right\|$. We denote by $\lambda_{k}(D)$ the weight of an optimum $k$-pack. Let $\mathcal{R}^{2}=\left\{R_{1}, R_{2}\right\}$ be the 2-pack of the digraph $D$ presented in Figure 1b. The weight of such 2-pack is 7 , since it covers all vertices except one. Note that such 2-pack is optimum since $\lambda(D)=4$ and every path of cardinality 4 has the same initial vertex. The dual version of Linial's Conjecture, stated below, was also stated in paper [Linial 1981].

Conjecture 3 (Linial's Dual). Let $D$ be a digraph and $k$ a positive integer. Then,

$$
\chi_{k}(D) \leq \lambda_{k}(D)
$$

It is not hard to see that when $k=1$ Linial's Dual Conjecture is exactly GallaiRoy's Theorem; therefore valid. Verify that, for the digraph $D$ depicted in Figure 1a, $\chi_{4}(D)=\chi_{3}(D)=|V(D)|=\lambda_{3}(D)=\lambda_{4}(D)$ and, according to our previous discussion, $7=\chi_{2}(D)<\lambda_{2}(D)=8$.

In order to dualize Berge's Conjecture, it is also necessary to redefine the concept of orthogonality. A coloring $\mathcal{C}=\left\{C_{1}, C_{2}, \ldots, C_{m}\right\}$ and a $k$-pack $\mathcal{P}^{k}$ are orthogonal if each color class $C_{i} \in \mathcal{C}$ meets $\min \left\{\left|C_{i}\right|, k\right\}$ distinct paths of $\mathcal{P}^{k}$. Note that, by definition of orthogonality, if $\mathcal{C}$ and $\mathcal{P}^{k}$ are orthogonal, then each color class of $\mathcal{C}$ meets as many paths as possible in $\mathcal{P}^{k}$. Berge's Dual Conjecture is stated below; it is, however, known to be false in general.

Conjecture 4 (Berge's Dual - false). For every k-optimum coloring $C$ of a digraph $D$, there is a k-pack orthogonal to $\mathcal{C}$.

For the particular example depicted in Figure 1b, Berge's Dual Conjecture holds. Note that for the 2-optimum coloring given the 2-pack $\mathcal{R}^{2}=\left\{R_{1}, R_{2}\right\}$ presented is orthogonal since each of $R_{1}$ and $R_{2}$ has one vertex of each color class of the coloring. In graphs for which Berge's Dual Conjecture is valid, Linial's Dual Conjecture must also hold. That is because $\lambda_{k}(D)$ is at least the size of the set of vertices covered by the $k$ pack orthogonal to the coloring, which in turn is exactly $\chi_{k}(D)$. However, Berge's Dual Conjecture is known to be false for arbitrary digraphs. A counterexample is depicted in Figure 2; three colors (white, black and gray) are necessary. An arbitrary coloring of this digraph with three colors is an 1-optimal coloring, but there is no path able to meet every color in the coloring shown on Figure 2.

On the other hand, it was not known so far whether the conjecture was true for bipartite digraphs. Note that the underlying undirected graph of the counterexample is an odd cycle, and bipartite digraphs are free of such structure. Moreover, Berge's Conjecture (Conjecture 2) is known to be valid for bipartite digraphs. Those observations motivated us to investigate Berge's Dual Conjecture for this particular class of digraphs.

It is also important to mention that, due to the falsehood of Berge's Dual Conjecture for general digraphs, Aharoni, Hartman, and Hoffman proposed 


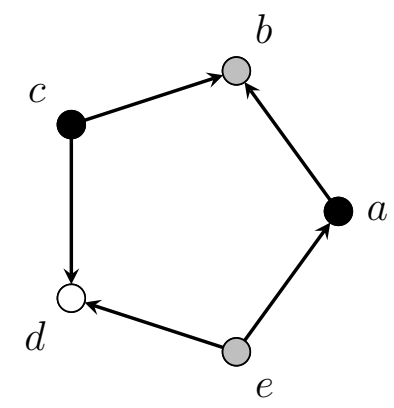

Figure 2. Berge's Dual Conjecture Counterexample $(k=1)$ - General Case

in [Aharoni et al. 1985] an alternative conjecture which also generalizes Linial's Dual Conjecture:

Conjecture 5 (Aharoni-Hartman-Hoffman). Let $D$ be a digraph and $k$ be a positive integer. Then for every optimum k-pack $\mathcal{P}^{k}$ there is a coloring $\mathcal{C}$ orthogonal to $\mathcal{P}^{k}$.

Aharoni-Hartman-Hoffman's Conjecture is also known to be valid for bipartite digraphs; the proof is presented in [Hartman et al. 1994]. Such result served as one more evidence for the potential validity of Berge's Dual Conjecture for bipartite digraphs.

A comprehensive survey on both primal and dual versions of these problems can be found in [Hartman 2006].

\section{Berge's Dual Conjecture for Bipartite Digraphs}

In this section, we show that for bipartite digraphs the problem of finding a $k$-pack orthogonal to the given optimal coloring reduces to the problem of finding a $k$-pack composed by a selection of $\min \left\{\alpha^{\prime}(D), k\right\}$ arcs of a maximum matching of $D$ together with $k-\alpha^{\prime}(D)$ unmatched vertices of $D$ which must be selected with some criteria. This implies that, in the bipartite case, the directions of the arcs are not relevant to the proof of Berge's Dual Conjecture. Moreover, the fact that the digraphs are bipartite, and, thus, admit a 2 -coloring, implies that for every coloring $\mathcal{C}$ the $k$-norm of $C$ is at most $2 k$, since each color class can count at most $k$. Then, $\chi_{k}(D) \leq 2 k$. Therefore, if there is a $k$-optimal coloring with two color classes of size at least $k$, then these two classes must be the only two in the $k$-optimal coloring.

Theorem 8. Let $D$ be a bipartite digraph and $\mathcal{C}$ a $k$-optimal coloring of $D$. Then, there is a k-pack $\mathcal{P}$ orthogonal to $\mathcal{C}$.

Proof. Let $\mathcal{C}=\left\{C_{1}, C_{2}, \ldots, C_{m}\right\}$ be a $k$-optimal coloring of $D$. We may assume, without loss of generality, that $\left|C_{1}\right| \geq\left|C_{2}\right| \geq \ldots \geq\left|C_{m}\right|$.

We will prove that there always is a $k$-pack $\mathcal{P}$ composed by a matching and unmatched vertices that meets $\min \left\{\left|C_{i}\right|, k\right\}$ vertices for each $C_{i}$. If $\mathcal{P}$ meets $\min \left\{\left|C_{i}\right|, k\right\}$ vertices for every $C_{i} \in \mathcal{C}$ the only missing restriction to claim that orthogonality holds is the assurance that each color class meets different paths. But since an arc in a matching can only connect vertices from different color classes and unmatched vertices form different trivial paths, this condition is immediately satisfied. Thus, $\mathcal{P}$ is a $k$-pack orthogonal to $\mathcal{C}$ whenever it has the described structure. 
Consider first the case in which $\alpha(D) \leq k$. In this case, $\min \left\{\left|C_{i}\right|, k\right\}=\left|C_{i}\right|$ for each $C_{i}$. Therefore, by definition of orthogonality, every vertex must be in some path of an orthogonal $k$-pack. Let $M$ be a maximum matching of $D$ and let $I$ be the set of vertices of $D$ not matched by $M$. If $\alpha^{\prime}(D)+|I| \leq k$, the arcs in $M$ together with the trivial paths with vertices of $I$ form the $k$-pack we are seeking. But this inequality is always valid since $n=2 \alpha^{\prime}(D)+|I|$ and, by Corollary 1 ,

$$
\begin{aligned}
\alpha^{\prime}(D)+\alpha(D) & =2 \alpha^{\prime}(D)+|I| \\
\alpha(D) & =\alpha^{\prime}(D)+|I| \\
& \leq k .
\end{aligned}
$$

We may thus assume that $\alpha(D)>k$. If $\left|C_{1}\right| \leq k$, then $\min \left\{\left|C_{i}\right|, k\right\}=\left|C_{i}\right|$ for each $C_{i}$ and $|\mathcal{C}|_{k}=V(D)$. In this case, an arbitrary coloring having a set of size $\alpha(D)$ as a color class has smaller $k$-norm; a contradiction. Thus, $\left|C_{1}\right|>k$. Consider first the case in which $n-\left|C_{1}\right| \leq k$. We will now argue that $\left|C_{1}\right|=\alpha(D)$ in this case. Assume the contrary, that is, $\left|C_{1}\right|<\alpha(D)$. Now let $\mathcal{C}^{\prime}=\left\{C_{1}^{\prime}, C_{2}^{\prime}, \ldots, C_{m^{\prime}}^{\prime}\right\}$ be some coloring of $D$ such that $\left|C_{1}^{\prime}\right|=\alpha(D)$. The $k$-norm of $\mathcal{C}^{\prime}$ is thus at most $k+n-\alpha(D)<k+n-\left|C_{1}\right|$, contradicting the fact that $\mathcal{C}$ is $k$-optimal. So, $\left|C_{1}\right|=\alpha(D)$. By Corollary $1, \alpha^{\prime}(D)=$ $n-\alpha(D)=n-\left|C_{1}\right| \leq k$. One maximum matching $M$ with $\alpha^{\prime}(D)$ arcs cannot have an arc with both ends in $V(D)-C_{1}$ since that would imply that $M$ would also have an arc with both ends in $C_{1}$, which is an independent set. Thus, every maximum matching $M$ has precisely one end in $C_{1}$ and the other end in $V(D)-C_{1}$. Since $\alpha^{\prime}(D) \leq k$ and every color class $C_{i}, i \neq 1$ has size at most $k$, a $k$-pack $\mathcal{P}$ composed by some maximum matching $M$ together with $k-\alpha^{\prime}(D)$ vertices of $C_{1}$ as trivial paths is orthogonal to $\mathcal{C}$.

Finally, consider the case in which $n-\left|C_{1}\right|>k$. Since $\chi_{k}(D) \leq 2 k$, we deduce that $\mathcal{C}$ has precisely two color classes, both of size larger than $k$ and $\chi_{k}(D)=2 k$. Then, an arbitrary subset of $k$ arcs of some maximum matching $M$ form a $k$-pack orthogonal to $\mathcal{C}$. It suffices thus to show that $\alpha^{\prime}(D) \geq k$. By Corollary $1, \alpha^{\prime}(D)=n-\alpha(D)$ and if $n-\alpha(D)<k$, then we may define a coloring $\mathcal{C}^{\prime}=\left\{C_{1}^{\prime}, C_{2}^{\prime}, \ldots, C_{m^{\prime}}^{\prime}\right\}$ of $D$ such that $\left|C_{1}^{\prime}\right|=\alpha(D)$ and whose $k$-norm would be smaller than $2 k$, a contradiction. Thus $\alpha^{\prime}(D) \geq k$ indeed. And in every case considered there is a $k$-pack $\mathcal{P}$ orthogonal to $\mathcal{C}$.

\section{Berge's Dual Conjecture for Perfect Digraphs}

After proving Berge's Dual Conjecture for bipartite digraphs we tried to find another class of digraphs for which the conjecture could also hold. The counterexample shown on Figure 2 already implies that the conjecture will not hold for arbitrary digraphs $D$ with $\chi(D)=3$ nor for those that contain one single odd cycle. We thus decided to investigate the validity of the conjecture for perfect digraphs as those are free of certain odd cycles such as that on Figure 2. Instead of proving, we found a counterexample for Berge's Dual Conjecture for perfect digraphs.

A clique $W \subseteq V(G)$ in a graph $G$ is a subset of vertices where for every pair of distinct vertices $u, v \in V(W), u$ and $v$ are adjacent. We denote by $\omega(G)$ the cardinality of a maximum clique in $G$. A graph $G$ is perfect if $\chi\left(G^{\prime}\right)=\omega\left(G^{\prime}\right)$ for every induced subgraph $G^{\prime}$ of $G$. In [Chudnovsky et al. 2006] a long standing conjecture, today 
known as the Strong Perfect Graph Theorem, was proved. The statement of the theorem, a characterization of perfect graphs, is given below:

Theorem 9 (Chudnovsky et al). A graph $G$ is perfect if and only if neither $G$ nor $\bar{G}$ contains an induced odd cycle with five or more vertices.

Graph $\bar{G}$ is the complement of $G$, i. e. $V(\bar{G})=V(G)$ and $E(\bar{G})=\{(u, v)$ : $(u, v) \notin E(G))\}$. Therefore, $G$ is perfect if and only if $\bar{G}$ is perfect. A digraph $D$ is perfect if its underlying undirected graph $G(D)$ is perfect. Thus, the Strong Perfect Graph Theorem guarantees that the digraph in Figure 2 will not be an induced subgraph of any perfect digraph.

It's easy to see that Berge's Dual Conjecture is valid for $k=1$ for perfect digraphs, as has already been noted by Berge in [Berge 1982a]:

Theorem 10 (Berge). Let $D$ be a perfect digraph, and let $\mathcal{C}$ be an 1-optimal (or simply optimal) coloring of $D$. Then, there is a path $P$ of $D$, orthogonal to $\mathcal{C}$.

Proof. Let $H$ be a maximum clique of $D$. We know that $\left|H \cap C_{i}\right|=1$ for every $C_{i} \in \mathcal{C}$. As $\mathcal{C}$ is an optimal coloring then $|H|=|\mathcal{C}|$. By Rédei's Theorem, there is a path $P$ that meets every color in $H$. Path $P$ is orthogonal to $\mathcal{C}$.

During our study of Berge's Dual conjecture for perfect digraphs we attempted a similar approach to the one used in the case of bipartite digraphs. The problem was divided in subcases based on different sizes of the maximum independent set. For the subcase when the size of the maximum independent set is less than or equal to $k$, Berge's Dual Conjecture does hold, as we show below.

Theorem 11. Let $D$ be a perfect digraph, and let $\mathcal{C}$ be a $k$-optimal coloring of $D$, for a positive integer $k$. If $\alpha(D) \leq k$, then there is a $k$-pack $\mathcal{P}_{k}$ of $D$ orthogonal to $\mathcal{C}$.

Proof. As $\alpha(D) \leq k$, then $\left|C_{i}\right| \leq k$ for every $1 \leq i \leq m$. As $D$ is perfect, so is $\bar{D}$. Thus, there is in $\bar{D}$ a coloring with $\chi(\bar{D})=\omega(\bar{D})=\alpha(D)$ colors. Each color class in $\bar{D}$ is a clique in $D$. So there is a partition of $D$ into $t=\alpha(D)$ cliques $\mathcal{H}=\left\{H_{1}, H_{2}, \ldots, H_{t}\right\}$. By Rédei's Theorem, there is a hamiltonian path in each $H_{i} \in \mathcal{H}$.

Let $\mathcal{P}_{t}$ be a $t$-pack composed by hamiltonian paths of each $H_{i} \in \mathcal{H}$. As $t=$ $\alpha(D) \leq k$, then $\mathcal{P}_{t}$ is a $k$-pack orthogonal to $\mathcal{C}$.

For the case when the maximum independent set has size greater than $k$, we found a counterexample for Berge's Dual Conjecture. Our counterexample is depicted in Figure 3.

The digraph $D$ in Figure 3 is perfect. It is easy to check that the size of the largest clique is always equal to its chromatic number for any subgraph $D^{\prime}$ induced by $D$. The 3 -coloring $\mathcal{C}$ of $D$ shown in the figure has 2-norm equal to 6 . As $D$ has 9 vertices and the size of its maximum independent set is 4 , then the only way to construct a coloring $\mathcal{C}^{\prime}$ with 2-norm smaller than 6 , would be by having a 3-coloring with two color classes with size $\alpha(D)$. However, it is not possible to have in $D$ two disjoint maximum independent sets. Thus, the coloring $\mathcal{C}$ is 2 -optimal. To find a 2-pack orthogonal to $\mathcal{C}$, it would be necessary to have two disjoint paths such that each path contained each one of the three colors, since every color class has size at least two. However, it is not possible to find two disjoint paths able to meet every color of this digraph. Then, there is no 2-pack orthogonal to $\mathcal{C}$. 


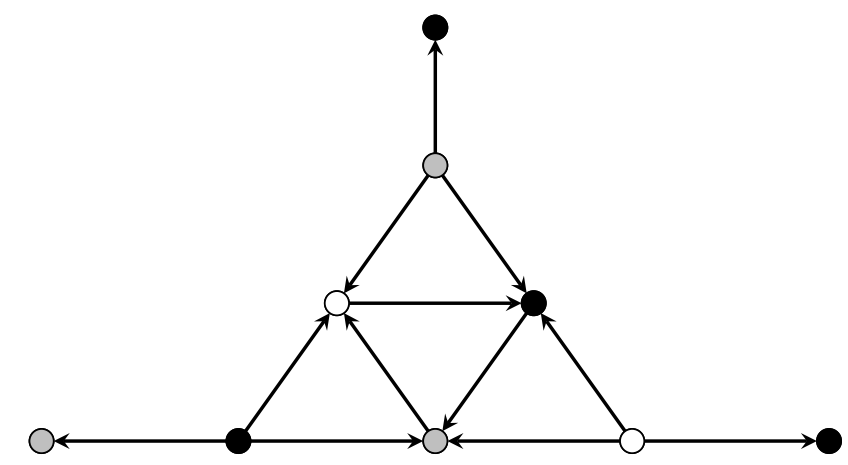

Figure 3. Berge's Dual Conjecture Counterexample $(k=2)$ - Perfect Digraphs

\section{Conclusion}

In this paper we showed that Berge's Dual Conjecture is valid for bipartite digraphs. Although the problem has been defined for directed graphs, the solution presented only uses trivial vertices and arcs of a maximum matching as paths to build the $k$-pack orthogonal to a given $k$-optimal coloring. The problem was thus reduced to a maximum matching problem where the orientation of the edges does not matter; a somewhat surprising fact. This proof of Berge's Dual Conjecture for bipartite digraphs presented in this paper has been published in the 4th edition of the Encontro de Teoria da Computação (Meeting of Computing Theory) of the XXXIX edition of the Congresso da Sociedade Brasileira de Computação (Congress of the Brazilian Computer Society) [Silva et al. 2019].

Furthermore, we presented an attempt to extend the result for perfect digraphs. Although Berge's Dual Conjecture is valid for some subcases, a counterexample was found. The existence of a counterexample for perfect digraphs suggests that it might be hard to understand what is the structure present in a digraph that prevents it from satisfying Berge's Dual Conjecture.

\section{Authors' Contribution}

Caroline Aparecida de Paula Silva was an undergraduate student at UFSCar until 2019. She was advised in this work, developed during a bit more than one year, by Cândida Nunes da Silva. Orlando Lee, professor at IC-UNICAMP, is a regular research partner of Cândida and the two have been collaborating in the past years researching several problems related to Berge's Dual Conjecture. The project was proposed to Caroline by both Cândida and Orlando, who shared the curiosity of figuring out if, at least for a so well structured class of digraphs such as bipartite digraphs, the Conjecture could hold. We did not expect to have a full answer to this question, given the lack of information regarding this specific Conjecture in the literature. Having an answer was quite a pleasant surprise. In the initial development of the project Orlando and Cândida had some discussions as to what would be the most promising strategy to attack the problem, how to best define the cases to be analyzed and which one seemed simpler and should be attacked first. These discussions where periodically reported to Caroline, who got deeply involved in the research. Very soon, Caroline started to examine some examples, trying to find some sort of algorithm to obtain the $k$-pack orthogonal to a given coloring. Her ideas, when presented to Cândida, resembled too much augmenting path algorithms for matching problems. At that moment Caroline was advised to study well known theorems and algorithms for max- 
imum matching in bipartite graphs so as to have more tools to attack the problem. So did Caroline. Not too long after that, Caroline figured out how to prove the Conjecture based, in essence, on Gallai's and König's classic theorems. Thus, Caroline deserves a lot of credit in this work.

Once the bipartite case was solved, it was published and presented at ETC-CSBC in 2019. There, Caroline was asked if the result could be extended to perfect digraphs or at least some subclass of those. There was no answer back then and, again, it did not seem it was possible to obtain an answer quickly. Even so, intense curiosity motivated us to at least start an investigation. To our surprise, we, again, obtained an answer quickly. This time, however, it is a negative answer.

\section{References}

Aharoni, R., Hartman, I. B.-A., and Hoffman, A. J. (1985). Path partitions and packs of acyclic digraphs. Pacific Journal of Mathematics, 2(118):249-259.

Berge, C. (1982a). Diperfect graphs. Combinatorica, 2(3):213-222.

Berge, C. (1982b). k-optimal Partitions of a Directed Graph. European Journal of Combinatorics, 3(2):97-101.

Chudnovsky, M., Robertson, N., Seymour, P., and Thomas, R. (2006). The strong perfect graph theorem. Annals of Mathematics, 164:51-229.

Dilworth, R. P. (1950). A decomposition theorem for partially ordered sets. Annals of Mathematics, 51(1):161-166.

Gallai, T. (1959). Über extreme punkt-und kantenmengen. Etvs Sect. Math, 2:133-138.

Gallai, T. (1968). On directed paths and circuits. Theory of graphs, pages 115-118.

Gallai, T. and Milgram, A. N. (1960). Verallgemeinerung eines graphentheoretischen Satzes von Rédei. Acta Sci Math, 21:181-186.

Hartman, I. B.-A. (2006). Berge's conjecture on directed path partitions-a survey. Discrete Mathematics, 306(19-20):2498-2514.

Hartman, I. B.-A., Saleh, F., and Hershkowitz, D. (1994). On Greene's theorem for digraphs. Journal of Graph Theory, 18(2):169-175.

König, D. (1931). Graphen und Matrizen. Matematikai Lapok, 38:116-119.

Linial, N. (1981). Extending the Greene-Kleitman theorem to directed graphs. Journal of Combinatorial Theory, Series A, 30(3):331-334.

Mirsky, L. (1971). A dual of Dilworth's decomposition theorem. Amer. Math. Monthly, 78:876-877.

Rédei, L. (1934). Ein kombinatorischer satz. Acta Litt. Szeged, 7(39-43):97.

Roy, B. (1967). Nombre chromatique et plus longs chemins d'un graphe. ESAIM: Mathematical Modelling and Numerical Analysis-Modélisation Mathématique et Analyse Numérique, 1(5):129-132.

Silva, C. A. P., Silva, C. N., and Lee, O. (2019). A proof for Berge's dual conjecture for bipartite digraphs. In Anais do IV Encontro de Teoria da Computação, Porto Alegre, RS, Brasil. SBC. 